\title{
High Electronic Conductance through Double-Helix DNA Molecules with Fullerene Anchoring Groups
}

\author{
Kathia L. Jiménez-Monroy, ${ }^{*}{ }^{\dagger}$ Nicolas Renaud, ${ }^{*}{ }^{\dagger}$ Jeroen Drijkoningen, $^{\dagger, \S}$ David Cortens, ${ }^{\dagger}$ \\ Koen Schouteden," Christian van Haesendonck, "Wanda J. Guedens, ${ }^{\dagger}$ Jean V. Manca, ${ }^{\dagger, \S}$ \\ Laurens D. A. Siebbeles, ${ }^{\ddagger \oplus}$ Ferdinand C. Grozema, ${ }^{\ddagger}$ and Patrick H. Wagner ${ }^{\dagger, \perp}$ \\ ${ }^{\dagger}$ IMO-IMOMEC, Hasselt University, Campus Diepenbeek, Wetenschapspark 1, 3590 Diepenbeek, Belgium \\ ${ }^{\ddagger}$ Department of Chemical Engineering, Delft University of Technology, Julianalaan 136, 2628 BL Delft, The Netherlands \\ ${ }^{\S}$ IMO \& X-LaB, Agoralaan Building D, 3590 Diepenbeek, Belgium \\ "Department of Physics and Astronomy, Solid-State Physics and Magnetism Section, and ${ }^{\perp}$ Department of Physics and Astronomy, \\ Soft-Matter Physics and Biophysics Section, KU Leuven, Celestijnenlaan 200D, 3001 Leuven, Belgium
}

\section{Supporting Information}

ABSTRACT: Determining the mechanism of charge transport through native DNA remains a challenge as different factors such as measuring conditions, molecule conformations, and choice of technique can significantly affect the final results. In this contribution, we have used a new approach to measure current flowing through isolated double-stranded DNA molecules, using fullerene groups to anchor the DNA to a gold substrate. Measurements were performed at room temperature in an inert environment using a conductive AFM technique. It is shown that the $\pi$-stacked B-DNA structure is conserved on depositing the DNA. As a result, currents in the nanoampere range were obtained for voltages ranging between $\pm 1 \mathrm{~V}$. These experimental results are supported by a theoretical model that suggests that a multistep hopping mechanism between delocalized domains is responsible for the long-range current flow through this specific type of DNA.

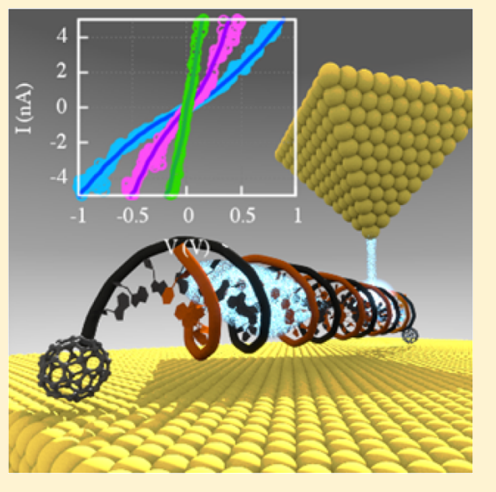

\section{INTRODUCTION}

The elucidation of the charge-transport mechanism along DNA is of prime interest for the design of nanoelectronic components $^{1-3}$ as well as for biological ${ }^{4,5}$ and medical applications. $^{6-10}$ Different experimental techniques such as photoinduced charge transfer ${ }^{11}$ or single molecule conductance experiments $^{12}$ can be used to probe the transfer of charges along a DNA molecule. In the latter the molecule is connected to macroscopic electrodes that are used to establish a steady state current flowing through the molecular structure. This can be achieved either using break junction techniques or by depositing the molecule on a substrate and probing its conductance using the tip of an STM or an AFM. Using such conductance techniques, a variety of electrical behaviors have been reported for DNA molecules, ranging from an insulator $^{13-16}$ to semiconductor ${ }^{17-22}$ or even a metal-like conductor. $^{23,24}$ These marked differences can partially be linked to the preservation of the double-helical B-DNA structure. It has been described that for DNA molecules deposited on a surface showing apparent heights of about $2.4 \mathrm{~nm}$ in AFM measurements behave as conductors. ${ }^{24}$ This height roughly corresponds to the diameter of B-DNA in which there is a relatively ordered $\pi$-stacked pathway for charge transport. In contrast, DNA molecules with an apparent height of about 1.1 $\mathrm{nm}$ behave like insulators, most likely due to the disruption of the B-DNA structure that diminishes the $\pi$-stack interactions between neighboring base pairs. An additional complication in comparing different conductance experiments is the nature of the anchoring groups that plays a key role in the overall electronic characteristics of DNA. ${ }^{25-27}$ These effects are partially due to their intrinsic electronic properties of the anchoring groups that dictate the energetics of the injection process. In addition, the way the anchoring groups connect the DNA molecule to the surface determines the magnitude of the electronic coupling to the electrode. These groups can for example be intercalated in the sequence or located at the end of the DNA molecule. Finally, the anchoring groups can also affect the integrity of the DNA structure, and the strength and geometry of the anchoring groups can either stabilize or destabilize the double-helical structure of the DNA and lead to large variations in the transport through the molecule itself.

Numerous theoretical models have been developed to simulate the transport of charges along DNA sequences. ${ }^{28-30}$ Early works suggested the existence of two distinct mechanisms-superexchange ${ }^{31-33}$ and incoherent hopping ${ }^{34}$ - to explain the charge transport along DNA molecules. According to these reports, the former is responsible of the charge transport along short DNA sequences containing up to three base pairs ${ }^{35}$ while the latter dominates the propagation of

Received: January 12, 2017

Published: January 17, 2017 
charge along longer sequences. ${ }^{36,37}$ Recently, the existence of intermediate charge transport mechanisms, mediated by either delocalized conduction channels ${ }^{34,38-43}$ or flickering resonances, ${ }^{44}$ has been proposed and to some extent observed experimentally. ${ }^{41,44-46}$ Despite their intrinsic differences, these recent models agree on the importance of thermal fluctuations within the DNA structure. ${ }^{47,48}$ These fluctuations facilitate charge propagation by introducing quasi-resonance between delocalized domains formed by a few strongly interacting base pairs. ${ }^{44}$

Previous attempts to probe the electronic behavior of DNA locally while preserving its native structure have proven to be challenging. ${ }^{27}$ We propose here an alternative way to study charge transport through single DNA molecules in their native structure by using fullerenes ${ }^{51}$ as anchoring groups in combination with conductive-AFM (C-AFM) at ambient temperature. The use of fullerene groups is interesting due to their small size, electronic properties, high affinity for gold, and possibility to be used in single molecule experiments. ${ }^{49-51}$ The strong binding of the fullerene to gold surface leads to strong electronic couplings that should improve the overall charge transfer mechanism. To our knowledge, this approach has not been reported before. Using this new technique, we observed long-range charge transport over more than $20 \mathrm{~nm}$ and with current intensities in the nanoampere range. The good transport properties of the DNA-fullerene (DNA-C61) complexes were rationalized using a kinetic model, where charge transport occurs via a hopping mechanism between active sites delocalized over four base pairs. These results suggest that DNA-C61 are interesting candidates for the realization of nanowires for future molecular electronic applications.

\section{EXPERIMENTAL METHODS}

Figure 1a shows an AFM topographic profile of a single DNAfullerene (DNA-C61) molecule which was deposited on an a)

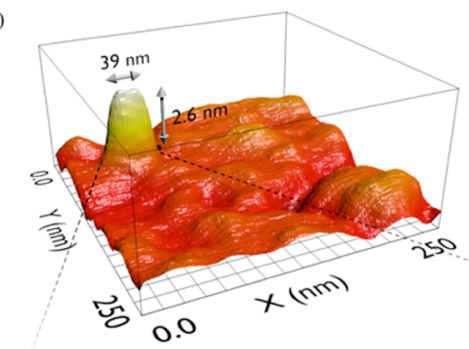

b)

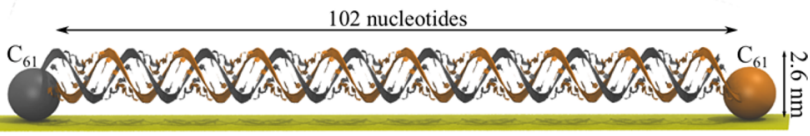

c)

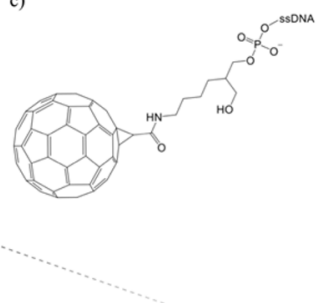

Figure 1. (a) Contact-AFM image of a single DNA-fullerene molecule on top of ultraflat gold (cantilever spring constant: $0.4 \mathrm{~N}$ / m; scan size: $0.25 \times 0.25 \mu \mathrm{m}^{2}$ ). (b) Schematic representation of the DNA-C61 molecule and (c) chemical structure of the C61-ssDNA binding group.

ultraflat gold surface. As can be seen in Figures $1 \mathrm{~b}$ and $1 \mathrm{c}$, the DNA-C61 molecules were obtained by the formation of an amide bond between carboxylic group of $\mathrm{C}_{60} \mathrm{CHCOOH}$ (C61) and the 3 '-position of amine-modified single-stranded DNA ( $\mathrm{NH}_{2}$-ssDNA) (102 nucleotides, $66.7 \%$ GC content). The specificity of the amide bond allows for the formation of only one C61 group attached to the $3^{\prime}$ end of one single-stranded
DNA. $^{52}$ The same procedure was repeated for the complementary $\mathrm{NH}_{2}$-ssDNA before hybridization of both strands.

After purification and characterization (Figures $\mathrm{S} 1-\mathrm{S} 3$ ), the double-stranded DNA-C61 molecules were deposited on ultraflat gold substrates (Platypus Technologies, LLC; Madison, WI) and dried for $24 \mathrm{~h}$ inside a desiccator (see Supporting Information for details). The GC content of the DNA sequence was intentionally designed to favor a G-hopping charge transfer mechanism, as suggested in the literature. ${ }^{53}$ Moreover, fullerenes are known to form better electrical contacts with gold than e.g. thiols and amines, ${ }^{50,51}$ hence allowing for C-AFM experiments at room temperature. ${ }^{51}$

Our synthesis protocol is based on the work of Shin et al., ${ }^{52}$ with minor changes. The compounds $\mathrm{C}_{60}-\mathrm{CHCOOH}$ (C61), $\mathrm{N}$-hydroxysuccinimide (NHS), and 1-ethyl-3-(3-(dimethylamino)propyl)carbodiimide (EDC) were purchased from Sigma-Aldrich (St. Louis, MO). The DNA sequences (ssDNA) were purchased from Eurogentec (Liège, Belgium). The exact sequence is reported in Supporting Information Section 1. After functionalization and removal of the unreacted compounds through dialysis, FT-IR spectroscopy (Bruker Tensor 27, Pike Miracle ATR cell) was used to verify that an amide group was formed between the fullerene and the DNA. The next step was to anneal and hybridize the two ssDNA strands. In order to verify the occurrence of only a single band of modified double-stranded DNA-C61 without impurities, the samples were analyzed on a $2 \%$ agarose gel with a reference DNA ladder, the GeneRuler 100 bp DNA ladder (Thermo Scientific Inc.). These DNA-C61 samples (0.1 mM PBS) were used for C-AFM and STM experiments. In order to obtain single molecules of DNA-C61, the original stock was diluted in Milli-Q water, drop-casted onto a flat gold surface, and dried with $\mathrm{N}_{2}$ flow, yielding $\sim 10$ molecules per $\mu \mathrm{m}^{2}$.

This sample was left in a desiccator for $24 \mathrm{~h}$ before performing analysis by either atomic force microscopy (CAFM, Bruker Multimode 8 AFM) or scanning tunneling microscopy (STM, Omicron Nano Technology, data shown in the Supporting Information). The C-AFM measurements were done inside a Faraday-shielded glovebox with protective $\mathrm{N}_{2}$ atmosphere at room temperature and relative humidity below $6 \%$. The STM measurements were performed at room temperature under ultrahigh vacuum (base pressure below 5 $\left.\times 10^{-11} \mathrm{hPa}\right)$.

\section{RESULTS AND DISCUSSION}

All AFM experiments were performed with a Pt-Ir AFM tip in a nitrogen environment (relative humidity $<6 \%$ ), using a Faraday-cage adaptation inside the AFM (Bruker Multimode 8 AFM) to avoid electronic disturbances. A control experiment was done using C61, solubilized in Milli-Q water and deposited in gold (Figures S4 and S5). For each AFM measurement, the total height $(2.6 \pm 0.1 \mathrm{~nm})$ and length $(39 \pm 1 \mathrm{~nm})$ were verified to correspond to an individual double-stranded DNAfullerene molecule (DNA-C61 of $102 \mathrm{bp}$ ) in its standard Bform and lying horizontally on the surface (see Figure 1a). ${ }^{24}$ Because of the drying procedure, one might assume that $B$ DNA can undergo a structural transition to A-DNA or to ZDNA in the absence of water. However, this would result in a compressed or a supercoiled structure, which was not observed in our experiments. ${ }^{24}$ This suggests that the low, but nonzero, humidity level in the AFM chamber is still sufficient to stabilize the $B$-conformation via hydrated counterions. ${ }^{54}$ 

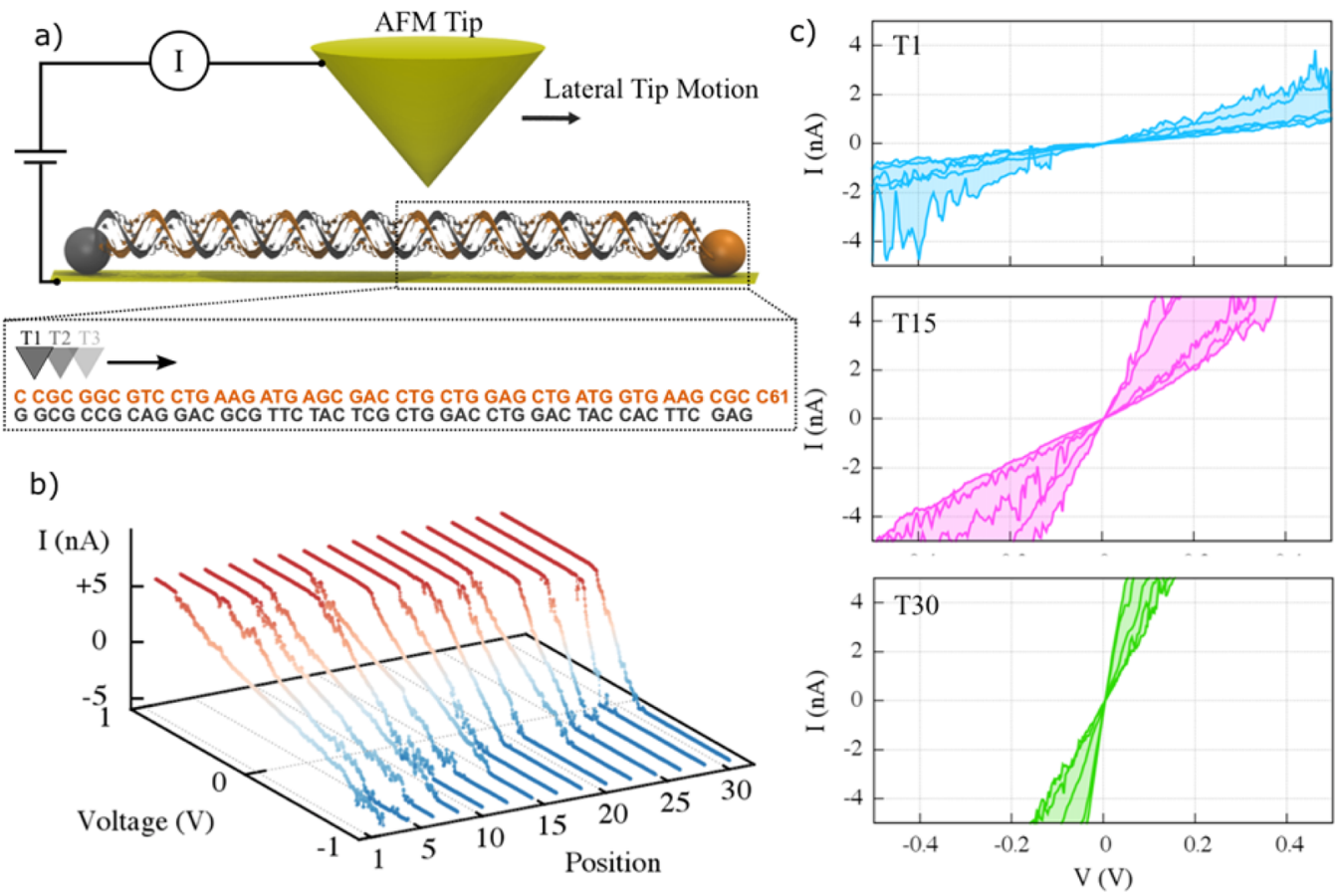

Figure 2. Schematic representation of the C-AFM experiments. (a) The Pt-Ir tip is brought on top of the DNA-C61 molecule at the starting position $\mathrm{T} 1$ (middle of the molecule) and voltages between -1 and $+1 \mathrm{~V}$ are applied. $I(V)$ curves are measured stepwise every $0.65 \mathrm{~nm}$. (b) Change of $I(V)$ curves when the AFM tip moves from T1 toward a fullerene end. The current cutoff at $\pm 5 \mathrm{nA}$ is due to signal saturation. (c) $I(V)$ curves obtained with five different molecules for three representative tip positions.

C-AFM measurements were performed by positioning the AFM tip in the middle of individual DNA-fullerene molecules and applying a voltage between the tip and the gold surface (see Figure $2 \mathrm{a})$. Voltage scans $(-1$ to $+1 \mathrm{~V})$ were done at 30 different positions on each molecule, starting from the middle and going toward one of the fullerene ends and finally out of the sample with a fixed translation step of $0.65 \mathrm{~nm}$. Figure $2 \mathrm{~b}$ shows the $I(V)$ curves measured at selected positions along a given molecule. As seen in this figure, we observed a clear steepening of the $I(V)$ slope when the tip was moved from its starting position (T1) in the middle of the molecule to the fullerene end (T30). The $I(V)$ slope at $\mathrm{T} 1$ corresponds to a resistance of $\sim 200 \mathrm{M} \Omega$ and decreases to $20 \mathrm{M} \Omega$ at position T30. We note that the apparent current cutoff at $\pm 5 \mathrm{nA}$ is due to the limited current range of the C-AFM setup. The complete set of curves can be found in the Supporting Information (Figure S5). We also found that the type of base pairs in direct contact with the AFM tip seems to affect the $I(V)$ slope. For regions rich in G-C base pairs, the $I(V)$ curves are almost linear while they present a typical semiconductor aspect in regions containing mainly A-T pairs (Figures S6, S7 and Table S1). In addition, we also investigated the structural effect of desalting the DNA-C61 molecules, which resulted in very low currents compared to the standard DNA-C61 molecules (Figures S8, S9 and Table S2).

Identical $I(V)$ measurements were performed on 22 different DNA-C61 molecules. Conductive behaviors similar to those shown in Figure $2 \mathrm{~b}$ were observed in 19 out of 22 of these samples. To illustrate the relative reproducibility of our experiment, we show in Figure $2 c$ the $I(V)$ curves of five different molecules measured at the selected positions $\mathrm{T} 1, \mathrm{~T} 15$, and T30. Although the measurements were taken over a period of several days and that there is a subnanometer uncertainty in hitting the exact starting position $\mathrm{T} 1$, the different measure- ment present the same trend as the conductance increases strongly when the tip approaches the terminal fullerene group.

The variation of the conductance observed in Figures $2 b$ and $2 c$ with respect of the tip position clearly indicates that the charge transport occurs along the entire length of the DNA strand and not in perpendicular direction between the C-AFM tip and the surface through individual base pairs. In addition, experiments performed on freshly prepared samples using a scanning tunneling microscope confirmed the absence of any type of vertical tunneling mechanism through these molecules (Figures S10 and S11). The possibility of direct charge tunneling between the AFM tip and the surface was also tested using coherent charge transport simulations (see Section 5 of the Supporting Information). These simulations show that the current intensities due to this mechanism would remain close to zero in the voltage range used here. This is due to the large band gap of the molecule and the very weak electronic couplings $(0.1-1 \mathrm{meV})$ between the molecular orbitals of the base pairs and the electronic states of the electrodes. Thus, charges are more likely to propagate through the $\pi$-stacked base pairs (Figures S12-S14), where electronic couplings are strong $(50-150 \mathrm{meV})$, rather than to tunnel from the tip to the surface (Figures S15-S17).

The large current intensities reported in Figure 2 confirm that the strategy employed here to deposit DNA-C61 on a gold surface preserves the B-DNA structure and does not disrupt the $\pi$-stacking between neighboring base pairs. An important point to note here is that as mentioned above, the shape of the $I(V)$ curve changes when the nature of the base pairs that is in direct contact with the AFM tip changes. This shows that the high currents observed here are due to transport through the DNA $\pi$-stack and transport via salt atoms adsorbed on the DNA can be ruled out. ${ }^{54}$ 

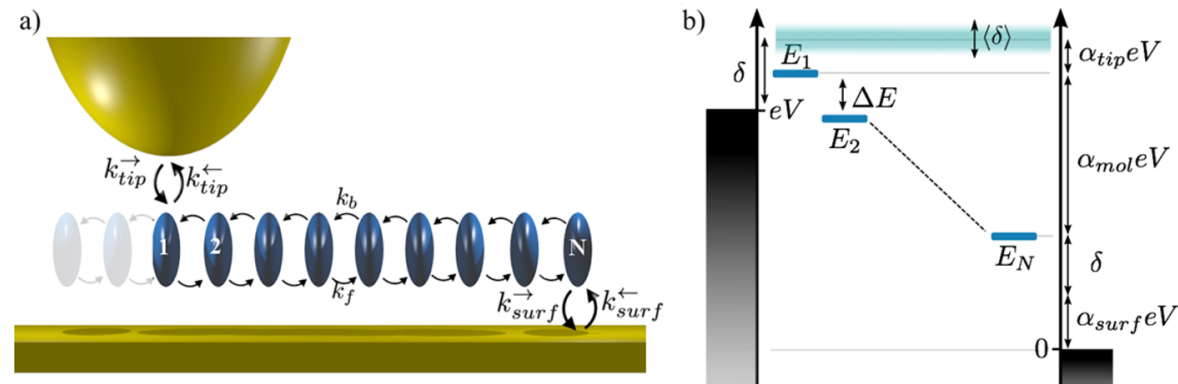

Figure 3. Representation of the kinetic charge-transport model. (a) The model involves multistep charge hopping between $N$ active sites, each of them corresponding to several interacting base pairs. The injection and extraction rates $k_{\text {tip/surf }}^{\rightarrow / \leftarrow}$ are here calculated following ref 38 . The inter-basepair rates $k_{\mathrm{b}}$ and $k_{\mathrm{f}}$ are calculated via a balance equation. (b) The voltage drop along the DNA is assumed to be linear.

As an additional confirmation of the direct relation between the conformation of the DNA and its conductive properties, we have also studied desalinated DNA-C61 molecules. In these experiments we observed considerably reduced conduction values (Figures S8, S9 and Table S2). This is as expected since desalination is accompanied by a disruption of the $B$-DNA structure and a poor $\pi$-stacking along the strand. ${ }^{15}$ Hence, the presence of ions and transport along the $\pi$ stack are indirectly related.

To understand the origin of the large current intensities observed experimentally, we have modeled the charge transport along the DNA using the rate equation proposed by Lehmann et al. ${ }^{38}$ Figure 3 illustrates the assumptions and parameters entering this model (see Section 4 of the Supporting Information).

As seen in Figure 3, charges move via a series of incoherent hopping steps through a sequence of neighboring active sites. An important assumption of the model is that in the absence of voltage drop all the sites present the same energy. This assumption, which might seem counterintuitive given the heteropolymeric sequence studied here, was made to provide the simplest possible picture of the charge transfer mechanism. Accounting for the exact sequence would require the use of a full quantum mechanical description of the system or the introduction of a large number of parameters in the model. The former is beyond the scope of this article, and we believe that the latter would only cloud the understanding of the charge transfer mechanism. The voltage profile along the molecule is a key ingredient of this model, as it defines the energy landscape for charge propagation. Following previous work, ${ }^{22}$ we assume that the applied voltage drops linearly between the two termini of the molecule. The voltage profile is then governed by three parameters: $\alpha_{\text {tip }}$ and $\alpha_{\text {surf }}$ define the voltage drops at the tip and at the surface while $\alpha_{\text {mol }}$ is the total voltage drop along the molecule. The conservation of voltage imposes that $\alpha_{\text {tip }}+\alpha_{\text {surf }}$ $+\alpha_{\mathrm{mol}}=1$. Therefore, the voltage dependence of the current is determined by the interfacial charge transfer rates between the metallic contacts and the molecule $k_{\text {tip } / \text { surf }}^{\rightarrow}$. Following Polizzi et al., ${ }^{39}$ the charge injection rate from the tip to the molecule is given by

$$
\begin{aligned}
& k_{\text {tip }}^{\rightarrow}(V)=c_{\text {tip }} \int_{-\infty}^{\infty} \mathrm{d} x \exp \left[-\left(x-\frac{\lambda+\delta+\alpha_{\text {tip }} e V}{k_{\mathrm{B}} T}\right)^{2}\right. \\
& \left.\quad \times\left(\frac{k_{\mathrm{B}} T}{4 \lambda}\right)\right]\left(1+\mathrm{e}^{x}\right)^{-1}
\end{aligned}
$$

In this equation, $c_{\text {tip }}$ represents the coupling strength at the tipmolecule interface and $\lambda$ is the reorganization energy corresponding to the charge injection process. The other injection rates have similar expressions, described in Section 4 of the Supporting Information. The forward and backward charge transfer rates between different active sites along the molecule are determined by a balance equation $k_{\mathrm{f}}=k_{0} \mathrm{e}^{-\left[\alpha_{\mathrm{mol}} V /\left(2(N-1) k_{\mathrm{B}} T\right)\right]}$ and $k_{\mathrm{b}}=k_{0} \mathrm{e}^{+\left(\alpha_{\mathrm{mol}} \mathrm{l} V /\left(2(N-1) k_{\mathrm{B}} T\right)\right)}$ where $k_{0}$ is the charge-transfer rate between neighboring sites in the absence of applied voltage. These voltage-dependent transfer rates are used as parameters to solve a kinetic model given by the equations

$$
\begin{aligned}
& \dot{P}_{1}=-\left(k_{\text {tip }}^{\leftarrow}+k_{\mathrm{f}}\right) P_{1}+k_{\mathrm{b}} P_{2}+k_{\text {tip }}^{\rightarrow} P_{\text {contact }} \\
& \dot{P}_{n}=-\left(k_{\mathrm{b}}+k_{\mathrm{f}}\right) P_{n}+k_{\mathrm{b}} P_{n-1}+k_{\mathrm{f}} P_{n+1} \\
& \dot{P}_{N}=-\left(k_{\text {surf }}^{\leftarrow}+k_{\mathrm{f}}\right) P_{N}+k_{\mathrm{f}} P_{N-1}+k_{\text {surf }}^{\rightarrow} P_{\text {contact }}
\end{aligned}
$$

This system of equations is solved in the steady state, i.e., $\dot{P}_{N}=$ 0 , and with the normalization condition $P_{1}+P_{2}+\ldots+P_{N}+$ $P_{\text {contact }}=1$. Once the steady state populations of the sites are calculated, the currents flowing through the tip and the surface are obtained by

$$
\begin{aligned}
& I_{\text {tip }}(V)=-e\left(k_{\text {tip }}^{\rightarrow} P_{\text {contact }}-k_{\text {tip }}^{\leftarrow} P_{1}\right) \\
& I_{\text {surf }}(V)=-e\left(k_{\text {surf }}^{\leftarrow} P_{N}-k_{\text {surf }}^{\rightarrow} P_{\text {contact }}\right)
\end{aligned}
$$

Note that the values of $I_{\text {tip }}(V)$ and $I_{\text {surf }}(V)$ are identical in the steady state limit. In its present form, this model can only simulate two-terminal systems, and therefore we considered only the charge propagation between the AFM tip and the closest fullerene terminus. Hence, the calculated total current intensity is possibly underestimated. Because of the strong electronic coupling between the fullerene and the surface (Figure S15), its orbitals are hybridized with the electronic states of the electrodes, making the last base pair of the sequence the final active site in the hopping model. Thus, the interfacial rates $k_{\text {surf }}^{\rightarrow / \leftarrow}$ represent the charge injection/extraction between the fullerene and the DNA. The electronic couplings supporting this mechanism are however weak due to the linker group binding the fullerene to the DNA backbone.

Previous reports suggest that the charge density can be delocalized over up to six base pairs during its propagation along the DNA sequence. ${ }^{22,34}$ Therefore, we consider that each active site in the model corresponds to a short sequence of $N$ neighboring base pairs. This delocalization reduces the static energy disorder of the active sites by averaging the energies of individual base pairs. 

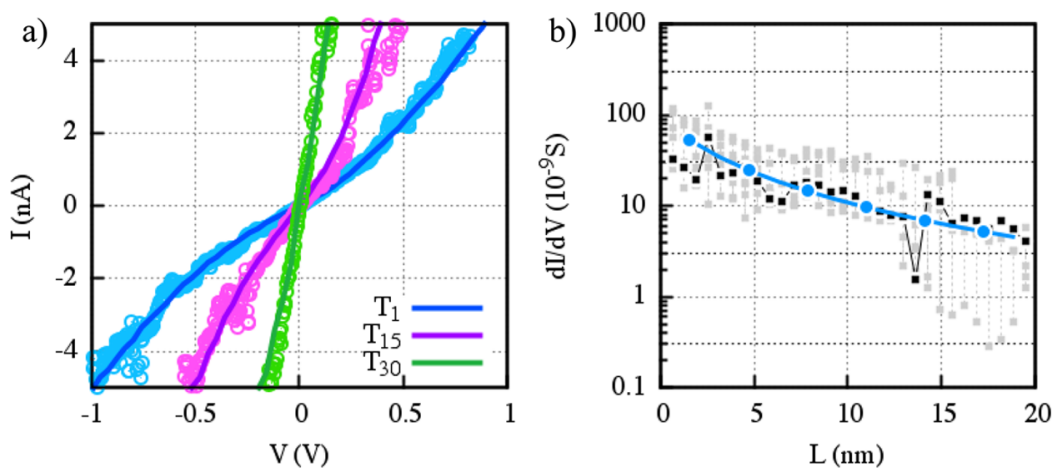

Figure 4. (a) Fit of $I(V)$ curves for a given DNA-C61 molecule at three different electrode positions using the kinetic model. The calculated curves (solid lines) are in good agreement with the experimental data (shown as circles). (b) Conduction variation of this molecule as a function of distance $L$ between the AFM tip and the terminal C61: Data are shown as black squares while the calculation is shown as dotted blue line. The gray square dots represent data for different DNA molecules at the same tip position along the double strand.

Table 1. Values of the Parameters Entering the Kinetic Model Fitted to the Experimental Data Shown in Figure $4 a^{a}$

\begin{tabular}{|c|c|c|c|c|c|c|c|c|c|}
\hline & $\delta(\mathrm{meV})$ & $\langle\delta\rangle(\mathrm{meV})$ & $\lambda(\mathrm{meV})$ & $c_{\text {tip }}\left(10^{10} \mathrm{~s}^{-1}\right)$ & $c_{\text {surf }}\left(10^{10} \mathrm{~s}^{-1}\right)$ & $\alpha_{\text {tip }}$ & $\alpha_{\text {surf }}$ & $k_{0}\left(10^{10} \mathrm{~s}^{-1}\right)$ & $N_{\text {site }}$ \\
\hline $\mathrm{T} 1$ & 65 & 25 & 85 & 10 & 10 & 0.1 & 0.2 & 23 & 13 \\
\hline $\mathrm{T} 15$ & 65 & 25 & 85 & 10 & 10 & 0.1 & 0.2 & 23 & 7 \\
\hline $\mathrm{T} 30$ & 65 & 25 & 85 & 10 & 10 & 0.1 & 0.2 & 23 & 1 \\
\hline
\end{tabular}

${ }^{a_{T}}$ The position T1 is model with 13 active sites but corresponds to 51 base pairs between the tip and the end of the molecule. This leads to about four base pairs per active site. Note that aside from the number of active sites, all the parameters are kept identical for the simulation of charge transport for different tip positions.

However, the disorder of DNA molecules at room temperature leads to fluctuations of these energies. Hence, we assume that the energies of the active sites are distributed following a normal distribution centered at $\delta$ (Figure $3 \mathrm{~b}$ ) with standard deviation $\langle\delta\rangle$. These fluctuations account for the slow modifications of the molecular structure. These modifications induce a change in the energy landscape that each electronic charge experiences during its transfer. The parameters entering the calculation of the current intensity (eq 3 ) were fitted to reproduce the experimental results displayed in Figure 2b. Since these experimental data were obtained for a single molecule, all model parameters were kept identical in the calculation with the exception of the tip position on the DNA-C61 complex. During the fitting procedure, the rate equation was solved $M=$ 250 times to average the effect of the static disorder. Characteristic fits are shown in Figure 4a, and the parameter values are summarized in Table 1 . Our numerical simulations indicate that each active site comprises $N=4$ neighboring base pairs, which is in good agreement with other recent results. ${ }^{22,34}$ The delocalization occurs probably within the blocks of identical neighboring base pairs contained in the sequence. Note that these blocks do not contain exactly 4 base pairs, and the delocalization length should be seen considered as an average size of these blocks.

As seen in Table 1 , the fit to the experimental data leads to an energy value for the active site of $\delta=65 \mathrm{meV}$. This value is relatively low compared to recent studies that lead for example to $\delta=550 \mathrm{meV}$ for $\mathrm{G}$ quadruplexes. ${ }^{22}$ However, the lack of band gap in the experimental $I(V)$ curves shown in Figure 2 clearly point at a low value of $\delta$. This value is a dictated by several parameters such as the nature of the electrodes and the structure of the molecule and therefore might vary significantly from one experiment to another. Our analysis also suggests that the static disorder is rather limited with a standard deviation $\langle\delta\rangle=25 \mathrm{meV}$. For comparison, other studies ${ }^{34,40-42}$ reported variations in the energies of the individual base pairs of up to
$100 \mathrm{meV}$. Note however that these previous estimates of the static disorder were obtained for a DNA hairpin in solution and not deposited on a rigid surface. This might explain the reduced static disorder observed in our experiment. The transfer rate between neighboring sites is here equal to $k_{0}=2.3 \times 10^{11} \mathrm{~s}^{-1}$. If we assume an inter-base-pair electronic coupling of $V_{\mathrm{bp}} \approx 50$ $\mathrm{meV}$ and a reorganization energy of $\lambda_{\mathrm{bp}} \approx 0.5 \mathrm{eV}$ as given by previous studies, ${ }^{47,48,56}$ this value of the inter-base-pair transfer rate is compatible with an adiabatic charge transfer mechanism between the two delocalized sites (see Supporting Information Section 6).

This relatively high value obtained for $k_{0}$ gives an additional confirmation that the structural integrity of the $B$-DNA is preserved in our experiment. A disruption of the $\pi$-stacking between neighboring base pairs would result in lower the charge transfer rates. Finally, the transfer rates $c_{\text {tip }}$ and $c_{\text {surf }}$ are both equal to $10 \times 10^{10} \mathrm{~s}^{-1}$, and the reorganization energy associated with charge injection is $85 \mathrm{meV}$. These values, combined with simple calculations of the electronic coupling between the AFM tip and the DNA reported in Figures S16 and $\mathbf{S 1 7}$, are consistent with a nonadiabatic charge transfer mechanism between the electrodes and the base pairs (see Supporting Information Section 6).

We have finally studied the distance dependence of the zerobias conductance $G=\mathrm{d} I /\left.\mathrm{d} V\right|_{V=0}$ obtained along the DNA-C61 molecules. The experimental values of conductance were directly extracted from the slope of the $I(V)$ curves measured for the 19 molecules and are reported in Figure $4 \mathrm{~b}$. The experimental data show a large spread but reveal a clear common trend as the conductance decreases with the distance between the tip and the terminal fullerene. Note that the deviations at distances exceeding $10 \mathrm{~nm}$ appear magnified due to the logarithmic scaling of the conductance axis. The values obtained for one specific molecule are highlighted by black squares. This shows the typical conductance variation observed during a single experiment. The theoretical curve of 
conductance vs distance was then calculated with the multistephopping model presented above using the values of the parameters reported in Table 1 . As seen in Figure $4 b$, there is a good agreement between theory and experiment as both show similar distance dependence. To confirm that charge transport occurs only along the DNA sequence, we have introduced a direct charge transport from each active site to the gold surface in our model (see Section 4 of the Supporting Information). As seen in Figures S13 and S14, such direct charge transfer leads to a much weaker distance dependence of the conductance and a pronounced nonlinearity of the $I(V)$ curves. Both are in contrast with the experimental results, which suggest that direct injection plays only a negligible role.

\section{CONCLUSIONS}

Our results indicate that DNA-C61 molecules are good candidates for the design of high-conductance nanowires. Our AFM characterization revealed that the structural integrity of the molecules was preserved during their deposition on the gold surface. The DNA then assumes its standard $B$-form with good $\pi$-stack interactions between neighboring base pairs. Conductive AFM measurements of the conductance of these complexes showed that DNA-C61 complexes are able to efficiently carry charges over large distances and with large current intensities. In addition, separate measurements on different molecules and on different days lead to similar results, illustrating the relative reproducibility of our results. These experimental findings were reinforced by a simple theoretical analysis based on a rate equation. These simulations indicate that an incoherent charge hopping mechanism could be responsible for the propagation of charges. The charges hop between identical active sites each containing four base pairs due to the charge delocalization. Despite its apparent simplicity compared to the complex sequence of the DNA molecule, this model was able to reproduce individual $I(V)$ curves and also lead to a distant dependence of the conductance that is in good agreement with our experimental data. The results presented in this article show that DNA-C61 molecules provide an attractive alternative for the production of nanowires that could be used as connections in electronic devices at ambient temperature.

\section{ASSOCIATED CONTENT}

\section{(S) Supporting Information}

The Supporting Information is available free of charge on the ACS Publications website at DOI: 10.1021/acs.jpca.7b00348.

Detailed description of the synthesis protocol and characterization techniques required to obtain single DNA-C61 molecules; theoretical charge transport models (tunneling and hopping) related to the experimental results (PDF)

\section{AUTHOR INFORMATION}

\section{Corresponding Authors}

*E-mail: jimenezmonroy.kathia@gmail.com (K.L.J.-M.).

*E-mail: n.renaud@tudelft.nl (N.R.).

\section{ORCID ${ }^{\circ}$}

Laurens D. A. Siebbeles: 0000-0002-4812-7495

Notes

The authors declare no competing financial interest.

\section{ACKNOWLEDGMENTS}

This project was funded by the Special Research Funds (BOF) of Hasselt University. The authors thank Dr. Patricia LosadaPérez and Dr. Alexander Volodin for fruitful discussions and Profs. Marlies K. Van Bael and Hans-Gerd Boyen for providing access to their experimental equipment.

\section{REFERENCES}

(1) Endres, R. G.; Cox, D. L.; Singh, R. R. P. Colloquium: The Quest for High Conductance DNA. Rev. Mod. Phys. 2004, 76, 195-214.

(2) Erler, C.; Günther, K.; Mertig, M. Photo-Induced Synthesis of DNA-Templated Metallic Nanowires and their Integration into MicroFabricated Contact Arrays. Appl. Surf. Sci. 2009, 255, 9647-9651.

(3) Yatsunyk, L. A.; Mendoza, O.; Mergny, J. L. 'Nano-oddities': Unusual Nucleic Acid Assemblies for DNA-Based Nanostructures and Nanodevices. Acc. Chem. Res. 2014, 47, 1836-1844.

(4) Bao, D.; Gao, P.; Wang, Y.; Zhou, H.; Chen, Y.; Chen, G.; Yang, P.; Zhang, X. Electrostatic Trapping of Double-Stranded DNA Based on $\mathrm{Cd}(\mathrm{OH})_{2}$ Three-Side Nanobelt Architectures. J. Phys. Chem. C 2015, 119, 1953-1959.

(5) Prado-Gotor, R.; Grueso, E. A Kinetic Study of the Interaction of DNA with Gold Nanoparticles: Mechanistic Aspects of the Interaction. Phys. Chem. Chem. Phys. 2011, 13, 1479-1489.

(6) Steenken, S. Purine Bases, Nucleosides, and Nucleotides: Aqueous Solution Redox Chemistry and Transformation Reactions of their Radical Cations and $\mathrm{e}^{-}$and $\mathrm{OH}$ Adducts. Chem. Rev. 1989, 89, 503-520.

(7) Breslin, D. T.; Coury, J. E.; Anderson, J. R.; McFail-Isom, L.; Kan, Y.; Williams, L. D.; Bottomley, L. A.; Schuster, G. B. Anthraquinone Photonuclease Structure Determines its Mode of Binding to DNA and the Cleavage Chemistry Observed. J. Am. Chem. Soc. 1997, 119, 50435044.

(8) Rajski, S. R.; Jackson, B. A.; Barton, J. K. DNA Repair: Models for Damage and Mismatch Recognition. Mutat. Res., Fundam. Mol. Mech. Mutagen. 2000, 447, 49-72.

(9) Merino, E. J.; Davis, M. L.; Barton, J. K. Common Mitochondrial DNA Mutations Generated through DNA-Mediated Charge Transport. Biochemistry 2009, 48, 660-666.

(10) Bhattacharya, P. K.; Barton, J. K. Influence of Intervening Mismatches on Long-Range Guanine Oxidation in DNA Duplexes. J. Am. Chem. Soc. 2001, 123, 8649-8656.

(11) Lewis, F. D.; Liu, X.; Liu, J.; Miller, S. E.; Hayes, R. T.; Wasielewski, M. R. Direct Measurement of Hole Transport in DNA. Nature 2000, 406, 51-53.

(12) Fink, H.-W.; Schönenberger, C. Electrical Conduction through DNA Molecules. Nature 1999, 398, 407-410.

(13) De Pablo, P. J.; Moreno-Herrero, F.; Colchero, J.; GómezHerrero, J.; Herrero, P.; Baró, A. M.; Ordejón, P.; Soler, J. M.; Artacho, E. Absence of DC-Conductivity in $\lambda$-DNA. Phys. Rev. Lett. 2000, 85, 4992-4995.

(14) Storm, A. J.; van Noort, J.; De Vries, S.; Dekker, C. Insulating Behavior for DNA Molecules between Nanoelectrodes at the $100 \mathrm{~nm}$ Length Scale. Appl. Phys. Lett. 2001, 79, 3881-3883.

(15) Zhang, Y.; Austin, R. H.; Kraeft, J.; Cox, E. C.; Ong, N. P. Insulating Behavior of Lambda-DNA on the Micron Scale. Phys. Rev. Lett. 2002, 89, 198102.

(16) Katsouras, I.; Piliego, C.; Blom, P. W. M.; de Leeuw, D. M. Transverse Charge Transport through DNA Oligomers in Large-Area Molecular Junctions. Nanoscale 2013, 5, 9882-9887.

(17) Porath, D.; Bezryadin, A.; de Vries, S.; Dekker, C. Direct Measurement of Electrical Transport through DNA Molecules. Nature 2000, 403, 635-638.

(18) Shapir, E.; Cohen, H.; Calzolari, A.; Cavazzoni, C.; Ryndyk, D. A.; Cuniberti, G.; Kotlyar, A.; Di Felice, R.; Porath, D. Electronic Structure of Single DNA Molecules Resolved by Transverse Scanning Tunnelling Spectroscopy. Nat. Mater. 2008, 7, 68-74.

(19) Watanabe, H.; Manabe, C.; Shigematsu, T.; Shimotani, K.; Shimizu, M. Single Molecule DNA Device Measured with Triple- 
Probe Atomic Force Microscope. Appl. Phys. Lett. 2001, 79, 24622464.

(20) Yoo, K.-H.; Ha, D. H.; Lee, J.-O.; Park, J. W.; Kim, J.; Kim, J. J.; Lee, H.-Y.; Kawai, T.; Choi, H. Y. Electrical Conduction through Poly(dA)-Poly(dT) and Poly(dG)-Poly(dC) DNA Molecules. Phys. Rev. Lett. 2001, 87, 198102.

(21) Lee, H.-Y.; Tanaka, H.; Otsuka, Y.; Yoo, K.-H.; Lee, J.-O.; Kawai, T. Control of Electrical Conduction in DNA Using Oxygen Hole Doping. Appl. Phys. Lett. 2002, 80, 1670-1672.

(22) Livshits, G. I.; Stern, A.; Rotem, D.; Borovok, N.; Eidelshtein, G.; Migliore, A.; Penzo, E.; Wind, S. J.; Di Felice, R.; Skourtis, S. S.; et al. Long-Range Charge Transport in Single G-quadruplex DNA Molecules. Nat. Nanotechnol. 2014, 9, 1040-1046.

(23) Murphy, C. J.; Arkin, M. R.; Jenkins, Y.; Ghatlia, N. D.; Bossmann, S. H.; Turro, N. J.; Barton, J. K. Long-Range Photoinduced Electron Transfer through a DNA Helix. Science 1993, 262, 10251029.

(24) Kasumov, A. Y.; Klinov, D. V.; Roche, P.-E.; Guéron, S.; Bouchiat, H. Thickness and Low-Temperature Conductivity of DNA Molecules. Appl. Phys. Lett. 2004, 84, 1007-1009.

(25) Hall, D. B.; Barton, J. K. Sensitivity of DNA-Mediated Electron Transfer to the Intervening $\pi$-stack: A Probe for the Integrity of the DNA Base Stack. J. Am. Chem. Soc. 1997, 119, 5045-5046.

(26) Chen, W.; Turro, C.; Friedman, L. A.; Barton, J. K.; Turro, N. J. Resonance Raman Investigation of $\mathrm{Ru}(\mathrm{phen})_{2}(\mathrm{dppz})^{2+}$ and Related Complexes in Water and in the Presence of DNA. J. Phys. Chem. B 1997, 101, 6995-7000.

(27) Heim, T.; Deresmes, D.; Vuillaume, D. Conductivity of DNA Probed by Conducting-Atomic Force Microscopy: Effects of Contact Electrode, DNA Structure, and Surface Interactions. J. Appl. Phys. 2004, 96, 2927-2936.

(28) Venkatramani, R.; Keinan, S.; Balaeff, A.; Beratan, D. N. Nucleic Acid Charge Transfer: Black, White and Gray. Coord. Chem. Rev. 2011, $255,635-648$.

(29) Berlin, Y. A.; Kurnikov, I. V.; Beratan, D.; Ratner, M. A.; Burin, A. L. In Long-Range Charge Transfer in DNA II; Schuster, G. B., Ed.; Springer: Berlin, 2004; pp 1-36.

(30) Genereux, J. C.; Barton, J. K. Mechanisms for DNA Charge Transport. Chem. Rev. 2010, 110, 1642-1662.

(31) Mallajosyula, S. S.; Pati, S. K. Toward DNA Conductivity: A Theoretical Perspective. J. Phys. Chem. Lett. 2010, 1, 1881-1894.

(32) Pemmaraju, C. D.; Rungger, I.; Chen, X.; Rocha, A. R.; Sanvito, S. Ab Initio Study of Electron Transport in Dry Poly(G)-Poly(C) ADNA Strands. Phys. Rev. B: Condens. Matter Mater. Phys. 2010, 82, 125426.

(33) Woiczikowski, P. B.; Kubar, T.; Gutiérrez, R.; Caetano, R. A.; Cuniberti, G.; Elstner, M. Combined Density Functional Theory and Landauer Approach for Hole Transfer in DNA Along Classical Molecular Dynamics Trajectories. J. Chem. Phys. 2009, 130, 215104.

(34) Renaud, N.; Berlin, Y. A.; Lewis, F. D.; Ratner, M. A. Between Superexchange and Hopping: An Intermediate Charge-Transfer Mechanism in Poly(A)-Poly(T) DNA Hairpins. J. Am. Chem. Soc. 2013, 135, 3953-3963.

(35) Roche, S. Sequence Dependent DNA-Mediated Conduction. Phys. Rev. Lett. 2003, 91, 108101.

(36) Berlin, Y. A.; Burin, A. L.; Ratner, M. A. Charge Hopping in DNA. J. Am. Chem. Soc. 2001, 123, 260-268.

(37) Bixon, M.; Jortner, J. Hole Trapping, Detrapping, and Hopping in DNA. J. Phys. Chem. A 2001, 105, 10322-10328.

(38) Lehmann, J.; Ingold, G. L.; Hänggi, P. Incoherent Charge Transport through Molecular Wires: Interplay of Coulomb Interaction and Wire Population. Chem. Phys. 2002, 281, 199-209.

(39) Polizzi, N. F.; Skourtis, S. S.; Beratan, D. N. Physical Constraints on Charge Transport through Bacterial Nanowires. Faraday Discuss. 2012, 155, 43-62.

(40) Felts, A. K.; Pollard, W. T.; Friesner, R. A. Multilevel Redfield Treatment of Bridge-Mediated Long-Range Electron Transfer: A Mechanism for Anomalous Distance Dependence. J. Phys. Chem. 1995, 99, 2929-2940.
(41) Segal, D.; Nitzan, A.; Davis, W. B.; Wasielewski, M. R.; Ratner, M. A. Electron Transfer Rates in Bridged Molecular Systems 2. A Steady-State Analysis of Coherent Tunneling and Thermal Transitions. J. Phys. Chem. B 2000, 104, 3817-3829.

(42) Xiang, L.; Palma, J. L.; Bruot, C.; Mujica, V.; Ratner, M. A.; Tao, N. Intermediate Tunnelling-Hopping Regime in DNA Charge Transport. Nat. Chem. 2015, 7, 221-226.

(43) Marcus, R. A.; Sutin, N. Electron Transfers in Chemistry and Biology. Biochim. Biophys. Acta, Rev. Bioenerg. 1985, 811, 265-322.

(44) Zhang, Y.; Liu, C.; Balaeff, A.; Skourtis, S. S.; Beratan, D. N. Biological Charge Transfer via Flickering Resonance. Proc. Natl. Acad. Sci. U. S. A. 2014, 111, 10049-10054.

(45) Renaud, N.; Berlin, Y.; Ratner, M. A. Impact of a Single Base Pair Substitution on Charge Transfer Rates Along Short DNA Hairpins. Proc. Natl. Acad. Sci. U. S. A. 2013, 110, 14867-71.

(46) Harris, M. A.; Mishra, A. K.; Young, R. M.; Brown, K. E.; Wasielewski, M. R.; Lewis, F. D. Direct Observation of the Hole Carriers in DNA Photoinduced Charge Transport. J. Am. Chem. Soc. 2016, 138, 5491-5494.

(47) Voityuk, A. A. Conformations of $\operatorname{Poly}(G)-\operatorname{Poly}(C) \pi$-stacks with High Hole Mobility. J. Chem. Phys. 2008, 128, 045104.

(48) Grozema, F. C.; Tonzani, S.; Berlin, Y. A.; Schatz, G. C.; Siebbeles, L. D. A.; Ratner, M. A. Effect of Structural Dynamics on Charge Transfer in DNA Hairpins. J. Am. Chem. Soc. 2008, 130, 51575166.

(49) Martin, C. A.; Ding, D.; Sørensen, J. K.; Bjørnholm, T.; van Ruitenbeek, J. M.; van der Zant, H. S. J. Fullerene-Based Anchoring Groups for Molecular Electronics. J. Am. Chem. Soc. 2008, 130, 13198-13199.

(50) Rogero, C.; Pascual, J. I.; Gómez-Herrero, J.; Baró, A. M. Resolution of Site-Specific Bonding Properties of C60 Adsorbed on $\mathrm{Au}(111)$. J. Chem. Phys. 2002, 116, 832-836.

(51) Leary, E.; González, M. T.; van der Pol, C.; Bryce, M. R.; Filippone, S.; Martín, N.; Rubio-Bollinger, G.; Agrait, N. Unambiguous One-Molecule Conductance Measurements Under Ambient Conditions. Nano Lett. 2011, 11, 2236-2241.

(52) Shin, S. R.; Jin, K. S.; Lee, C. K.; Spinks, G. M.; So, I.; Jeon, J.H.; Kang, T. M.; Mun, J. Y.; Han, S.-S.; Ree, M.; et al. Fullerene Attachment Enhances Performance of a DNA Nanomachine. Adv. Mater. 2009, 21, 1907-1910.

(53) Giese, B. Long-Distance Electron Transfer through DNA. Annu. Rev. Biochem. 2002, 71, 51-70.

(54) Kubař, T.; Gutiérrez, R.; Kleinekathöfer, U.; Cuniberti, G.; Elstner, M. Modeling Charge Transport in DNA Using Multi-Scale Methods. Phys. Status Solidi B 2013, 250, 2277-2287.

(55) Xu, B.; Zhang, P.; Li, X.; Tao, N. Direct Conductance Measurement of Single DNA Molecules in Aqueous Solution. Nano Lett. 2004, 4, 1105-1108.

(56) Siriwong, K.; Voityuk, A. A.; Newton, M. D.; Rösch, N. Estimate of the Reorganization Energy for Charge Transfer in DNA. J. Phys. Chem. B 2003, 107, 2595-2601.

(57) Liu, C.; Xiang, L.; Zhang, Y.; Zhang, P.; Beratan, D. N.; Li, Y.; Tao, N. Engineering Nanometer-Scale Coherence in Soft Matter. Nat. Chem. 2016, 8, 941-945.

(58) Renaud, N.; Harris, M. A.; Singh, A. P. N.; Berlin, Y.; Ratner, M. A.; Wasielewski, M. R.; Lewis, F. D.; Grozema, F. C. Deep-Hole Transfer Leads to Ultrafast Charge Migration in DNA Hairpins. Nat. Chem. 2016, 8, 1015-1021. 